MTX mono-therapy, combination therapy with MT +adalimumab $40 \mathrm{mg} \mathrm{s} / \mathrm{c}$ eow was started. At baseline and after 1 year. of therapy the following PROs were analysed: trends in fatigue (according to FACIT) and in RAPID3 were studied in 64 pts, trends in patient's global disease activity (PGA) and in patient's pain VAS were studied in 78 pts, trends in levels of anxiety and depression (HADS) were studied in 49 pts. The data acquired was analysed with the use of Spearman's correlation.

Results: After 1 year of therapy, there was a significant improvement in the following scores: anxiety changed from $5.7 \pm 3.1$ to $4.3 \pm 3.2$, $(p=0.003)$, fatigue from $35.3 \pm 9.6$ to $41.3 \pm 9.9(p<0.001)$, RAPID3 from $13.4 \pm 5.1$ to $6.2 \pm 5.2(p<0.001)$, PGA from $56.0 \pm 17.8$ to $18.9 \pm 17.1$ ( $p<0.001$ ), pain from $53.7 \pm 18.6$ to $16.9 \pm 16.6$ $(p<0.001)$. Depression scores had also changed though not significantly: from 3.8 \pm 3.0 to $3.2 \pm 3.1(p=0.235)$. The dynamics of anxiety and depression indexes correlated with the dynamics of fatigue ( $r=0.64$ and $r=0.39$, accordingly), as well as dynamics of FACIT and RAPID3 indexes $(r=0.36)$. Correlation of the dynamics of RAPID3 indexes with DAS $(r=0.45)$ and DAS28 $(r=0.41)$ activity reduction was found. Association of RAPID3 dynamics with the achievement of remission according to DAS $(p<0.001)$ and DAS28 $(p<0.001)$ was detected. Interrelation between RAPID3 dynamics and the achievement of MDA $(p<0.001)$ was found. Correlation between dynamics of anxiety and depression indexes and the reduction of tender joint count (TJC) ( $r=0.38$ and $r=0.36$, accordingly) was found. There is correlation between the dynamics of fatigue indexes and TJC, swollen joint count (SJC) and PGA ( $r=0.30, r=0.25$ and $r=0.35$, accordingly). Dynamics of RAPID3 correlated with TJC and SJC dynamics ( $r=0.33, r=0.25)$, as well as with $P G A$ and pain dynamics $(r=0.49$ and $r=0.58)$. $P G A$ and pain dynamics correlated with TJC and SJC dynamics ( $r=0.34$ and $r=0.26 ; r=0.43$ and $r=0.39$, accordingly). Conclusions: The T2T strategy in the Russian cohort of peripheral early PsA pts demonstrated the improvement of PROs indexes and decrease in PsA activity. Interrelation between the improvement of psychological status according to PROs (anxiety, depression and fatigue) and improvement in joint status (TJC and SJC) was found. RAPID3 is a reliable tool for evaluating patient's status: RAPID3 indexes correlate with the achievement of MDA and DAS/DAS28 remission.

Disclosure of Interest: None declared

DOI: 10.1136/annrheumdis-2018-eular.2413

\section{AB0919 VALIDATION OF PROTEOMIC BIOMARKERS OBSERVED IN MONOZYGOTIC TWINS CONFIRMS TWO PROTEINS ASSOCIATED WITH PSORIATIC DISEASE}

E. Generali ${ }^{1}$, N. Isailovic ${ }^{1}$, A. Ceribelli ${ }^{1}$, F. Sacrini ${ }^{2}$, M. De Santis ${ }^{1}$, M. Meroni ${ }^{1}$, G. M. Guidelli ${ }^{1}$, M. Caprioli ${ }^{1}$, A. Costanzo ${ }^{2,3}$, C. Selmi ${ }^{1,4}$. ${ }^{1}$ Rheumatology and Clinical Immunology; ${ }^{2}$ Dermatology Unit, Humanitas Research Hospital; ${ }^{3}$ Dermatology, Hunimed University, Rozzano; ${ }^{4}$ BIOMETRA Department, University of Milan, Milan, Italy

Background: Skin psoriasis affects $3 \%$ of the general population and as much as $20 \%-40 \%$ of patients will develop psoriatic arthritis (PsA), with the two conditions representing a clinical and immunological continuum within a prototype for chronic inflammation. Different from most rheumatic diseases, no serum autoantibody is associated with PsA, and there are currently no biomarkers for an early diagnosis and prediction of PsA onset in psoriasis, frequently causing a delayed diagnosis. Monozygotic twins discordant for psoriasis/PsA represent a unique setting to investigate the influence of environmental and stochastic factors on disease phenotype. Through a high-throughput proteomic analysis (SomaLogic) we have previously identified a set of 13 proteins differentially expressed in the serum of monozygotic twins discordant for psoriatic disease.

Objectives: To validate serum proteomic biomarkers of psoriatic disease using commercially available ELISA in monozygotic twins and in unrelated patients with psoriasis or PsA.

Methods: Our study included sera from our cohort of monozygotic twins previously described and from 70 unrelated patients with psoriatic disease (psoriasis without PsA $34 \%-49 \%$, PsA with/without psoriasis 36\%-51\%; women $46 \%$, median age 52 years, interquartile range 41-59) followed at Humanitas Research Hospital, and 25 healthy subjects ( $52 \%$ women, median age 52 years, IQR 4462). Candidate serum proteomic biomarkers obtained by SomaLogic analysis were validated using commercially available ELISA kits and proteins are herein anonymized due to a pending patent request.

Results: We found a significant correlation between SomaLogic results in monozygotic twins and ELISA results in unrelated psoriatic cases in the serum levels of 2 proteins (figure 1), which are involved in inflammatory and immune response, and one has been previously reported in psoriatic plaques. Four proteins showed a significantly different expression between psoriasis and PsA versus controls, in particular two proteins have a potential role in disease pathogenesis, as protein \#1 acts as cell-surface receptor and regulates differentiation, proliferation and survival of dendritic cells, while protein \#2 is involved in regulation of UV radiationinduced apoptosis and protein folding.
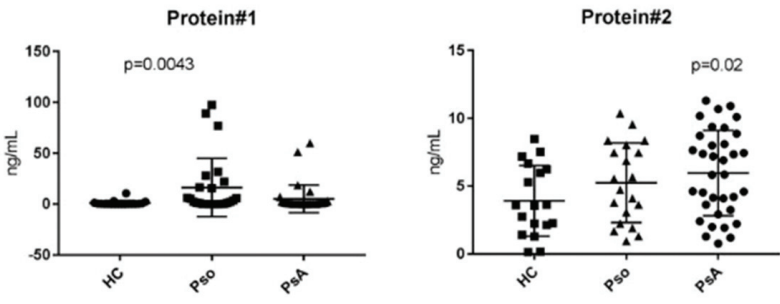

Abstract AB0919 - Figure 1

Conclusions: Two serum proteomic biomarkers, previously identified in a cohort of monozygotic twins discordant for psoriatic disease, can discriminate psoriatic disease, thus representing potential biomarkers of disease and possibly playing a pathogenetic role in disease.

Disclosure of Interest: None declared

DOI: 10.1136/annrheumdis-2018-eular.6250

\section{AB0920 1 TUMOUR NECROSIS FACTOR INHIBITORS PERSISTENCE IN PSORIATIC ARTHRITIS PATIENTS}

E. Vieira-Sousa ${ }^{1}$, M. Eusébio ${ }^{2}$, P. Ávila-Ribeiro ${ }^{1}$, N. Khmelinskii ${ }^{1}$, A.R. Machado ${ }^{1}$, T. Martins Rocha ${ }^{3}$, M. Bernardes ${ }^{3}$, D. Santos-Faria ${ }^{4}$, J. Leite Silva ${ }^{4}$, H. Santos ${ }^{5}$,

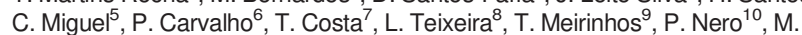
J. Santos ${ }^{8} .{ }^{1}$ Rheumatology and Metabolic Bone Diseases Department, Hospital de Santa Maria, CHLN and Rheumatology Research Unit, Instituto de Medicina Molecular, Faculty of Medicine, University of Lisbon, Lisbon Academic Medical Centre; ${ }^{2}$ Portuguese Society of Reumatology, Lisbon; ${ }^{3}$ Rheumatology Department, Centro Hospitalar São João do Porto and Faculdade de Medicina da Universidade do Porto, Porto; ${ }^{4}$ Rheumatology Department, Unidade Local de Saúde do Alto Minho, Ponte de Lima; ${ }^{5}$ Instituto Português de Reumatologia, Lisbon;

${ }^{6}$ Rheumatology Department, Hospitais Universitários de Coimbra, Coimbra;

${ }^{7}$ Rheumatology Department, Hospital de Egas Moniz, CHLO, Lisbon;

${ }^{8}$ Rheumatology Department, Hospital Garcia de Orta, Almada; ${ }^{9}$ Rheumatology Department, Centro Hospitalar do Baixo Vouga, Aveiro; ${ }^{10}$ Hospital CUF Descobertas, Lisbon, Portugal

Background: Tumour necrosis factor inhibitors (TNFi) lead to a dramatic improvement in the management of psoriatic arthritis (PsA). Nevertheless, a sig nificant proportion of patients still do not respond and/or are intolerant to TNFis, requiring treatment switch for an adequate control of disease activity.

Objectives: To assess TNFis drug retention and the main reasons for TNFi dis continuation in PsA patients.

Methods: This was a non-interventional study of PsA patients registered at the Rheumatic Diseases Portuguese Registry (Reuma.pt), with at least one TNFi prescription. Drug retention for a first, second and third line TNFi was assessed by Kaplan-Meier survival analysis. The reasons for discontinuation were described as frequencies.

Results: 750 PsA patients were included, with a mean age of 47.6 years $( \pm 11.6)$; $50.3 \%(n=377)$ female. 200 patients $(26.7 \%)$ treated with adalimumab, 335 $(44.7 \%)$ with etanercept, $114(12.2 \%)$ with golimumab and $101(13.5 \%)$ with infliximab, as first line TNFi. The majority $(67.6 \%)$ were receiving concomitantly conventional synthetic disease modifying anti-rheumatic drugs $(62.3 \% \mathrm{MTX})$ and $33.9 \%$ corticosteroids. The mean duration of TNFi retention was of $48.5 \pm 40.1$ months, when treated with a 1st TNFi, decreasing to $35.5 \pm 33$ months for the 2nd $\mathrm{TNFi}$, and to $22.7 \pm 22.9$ months for the $3 \mathrm{rd}$ TNFi (figure 1). After being treated with a 1st TNFi, the majority of discontinuers (35,8\% of the total population), withdraw due to lack or loss of effectiveness (53.5\%) and due to adverse events $(24.4 \%)$ The rates of discontinuation for the 2 nd and 3 rd TNFi were of $39 \%$ and $54 \%$, respectively. Lack or loss of effectiveness and adverse events were maintained the two main reasons of withdrawal for the 2 nd $(62.3 \% ; 21.6 \%)$ and 3 rd TNFi (63\%; 22.2\%). 